Relations industrielles

Industrial Relations

\title{
Note sur la grève tournante
}

\section{Louis LeBel et Pierre Verge}

Volume 25, numéro 1, 1970

URI : https://id.erudit.org/iderudit/028106ar

DOI : https://doi.org/10.7202/028106ar

Aller au sommaire du numéro

\section{Éditeur(s)}

Département des relations industrielles de l'Université Laval

ISSN

0034-379X (imprimé)

1703-8138 (numérique)

Découvrir la revue

\section{Citer cet article}

LeBel, L. \& Verge, P. (1970). Note sur la grève tournante. Relations industrielles / Industrial Relations, 25(1), 127-131. https://doi.org/10.7202/028106ar
Résumé de l'article

Une forme nouvelle de grève est apparue au Québec : la " grève tournante », ou arrêt de travail concerté et partiel, exécuté successivement par des ensembles de salariés compris dans une même unité de négociation. Juridiquement, ce procédé doit-il être reçu avec une faveur particulière, en tant qu'instrument de « civilisation » de la grève ? Faut-il, au contraire, y voir une forme particulièrement pernicieuse de l'exercice du droit de grève ?
Tous droits réservés (C Département des relations industrielles de l'Université Laval, 1970
Ce document est protégé par la loi sur le droit d'auteur. L'utilisation des services d'Érudit (y compris la reproduction) est assujettie à sa politique d'utilisation que vous pouvez consulter en ligne.

https://apropos.erudit.org/fr/usagers/politique-dutilisation/ 


\title{
DROIT DU TRAVAIL
}

\section{Note sur la grève tournante}

\author{
Louis LeBel \\ et \\ Pierre Verge
}

Une forme nouvelle de grève est apparue au Québec: la "grève tournante 》, ou arrêt de travail concerté et partiel, exécuté successivement par des ensembles de salariés compris dans une même unité de négociation. Juridiquement, ce procédé doit-il être reçu avec une faveur particulière, en tant qu'instrument de «civilisation》 de la grève? Faut-il, au contraire, $y$ voir une forme particulièrement pernicieuse de l'exercice du droit de grève?

Avant de tenter de préciser le régime juridique de la grève tournante, il nous faut, compte tenu de sa nouveauté, en préciser la nature.

\section{NOTION DE GRÈVE TOURNANTE}

Comme toute grève, la grève tournante se doit d'être une «cessation concertée de travail par un groupe de salariés $\gg 1$. Partant, on doit notamment la distinguer du «ralentissement d'activités 》(grève perlée), prohibé par le Code du travail ${ }^{2}$. Un jugement récent, traitant de la légalité de la grève tournante dans le secteur scolaire ${ }^{3}$ reconnaît bien cette distinction.

1. Code du travail, S.R.Q., 1964, ch. 141, a. 1, h).

2. Ibidem, a. 96.

3. Commissaires d'écoles pour la municipalité de Saint Tharcicius v. Association des enseignants de la Matapédia, C.S. (Q.) no 155-519, jugement du juge P. Côté en date du 5 mars 1969. (Des jugements identiques avaient été rendus le même jour, par le même juge dans quatre autres dossiers. Ces jugements avaient été portés en appel; depuis un règlement hors cour est intervenu.) 
Utilisant ici les termes d'auteurs français, qui, dans un contexte différent, ont analysé, depuis quelques années la grève tournante ${ }^{4}$, l'on peut dire que celle-ci se rattache au genre des grèves intermittantes avec cette particularité que les arrêts de travail qui la composent touchent successivement dans l'entreprise des catégories différentes de salariés : "Les grèves tournantes sont des grèves à exécution catégorielle et successive ${ }^{5}$. Quant à ces secteurs, dans l'entreprise, où, à divers moments, la grève s'exécute, observons qu'il peut s'agir de secteurs définis en fonction du lieu de travail ou encore de la profession des grévistes (grève tournante horizontale ou verticale, selon des auteurs français ${ }^{6}$ ). Transposée en droit québécois, la grève tournante, peut-on dire, mobilise successivement des salariés se rattachant à différents secteurs d'une même unité de négociation.

Cette forme de grève présente normalement des avantages certains pour ceux qui l'organisent et l'exécutent : diminution globale du rendement du travail avec maintien correspondant des frais généraux de l'employeur ; limitation de la perte totale de salaire chez les grévistes. Quant à ceux qui subissent la grève (employeurs et tiers), le fait qu'elle ne s'exécute que partiellement, à un même moment, améliorerait-il leur situation, dans une certaine mesure? Dans l'affirmative, ce facteur pourrait influer sur l'appréciation de la légalité de la grève tournante.

\section{RÉgIME JURIDIQUe DE LA GRÈve TOURNANTE}

La légalité de la grève tournante, comme celle de toute autre grève d'ailleurs, s'apprécie en regard, à la fois du droit commun et du Code du travail.

\section{LÉGALITÉ EN DROIT COMMUN}

Le droit de grève est acquis ? . Or la grève tournante a déjà été qualifiée de grève. Elle possède donc, en principe, le caractère de légalité. S'il $\mathrm{y}$ avait illégalité, il faudrait la rechercher dans son exercice.

4. De façon générale, en droit français, voir notamment les analyses suivantes de la grève tournante : $\mathrm{H}$. Sinay, La grève, t. 6 du Traité de droit du travail, publié sous la direction de G.H. Camerlynck, Paris, Dalloz, pp. 164 à 169, no 77 et suivants ; pp. 388 et suivantes, no179 et suivants; A. Brun et H. Galland, Droit $d u$ travail, mise à jour 1968, Paris, Sirey, 1968, pp. 295 et suivantes; A. Brun, La jurisprudence en droit du travail, Paris, Sirey, 1967, pp. 625 et suivantes.

5. H. Sinay, ibidem, p. 35 .

6. A. Brun et H. Galland, ibidem, p. 296.

7. Voir notamment $R$. Lachapelle, «Le contrôle de l'exercice du droit de grève au Québec 》, pp. 208 et sq. dans Travaux du quatrième colloque international de droit comparé, Ottawa, Presses de l'U. d'Ottawa, 1967. 
Le droit civil reconnait, en effet, que l'exercice d'un droit, peut, dans certains cas, devenir abusif, et, partant, devenir illicite ${ }^{8}$. L'on est alors le plus souvent en présence de l'exercice malicieux d'un droit, en l'absence de tout intérêt réel chez son titulaire. En d'autres termes, l'exercice de ce droit se trouve détourné de sa finalité.

Cela évidemment nous ramène à une situation d'espèce à analyser. Remarquons simplement ici que le juge Côté dans l'affaire précitée, sans en faire un motif de son jugement, se déclarait d'avis que les instituteurs avaient eu recours à la grève tournante dans le but «... de causer le plus d'ennuis possible à la Commission Scolaire sans faire supporter par les membres de l'Association intimée les ennuis pécuniaires et autres qu'une grève totale entrainerait 9 ». À l'inverse, la Commission des relations du travail fut témoin, dans une autre affaire, d'un recours à la grève tournante par des salariés d'un service public dans le but, selon le syndicat, d'éviter aux usagers les inconvénients d'une grève totale ${ }^{10}$.

L'approche du droit français en la matière peut éclairer le principe de la légalité de la grève tournante, mais retient la possibilité d'un exercice illégal de celle-ci, en raison d'un usage « abusif $», 11$ voire « excessif $» 12$. Les auteurs reconnaissent toutefois la difficulté d'appréciation de la légalité de cette forme de grève : pour être efficace, la grève doit être nocive ! $\mathrm{Ce}$ que l'on condamne, c'est la désorganisation de l'entreprise, voulue comme telle par les grévistes : distinction selon que les grévistes demeurent sur les lieux du travail ou non (dans l'affirmative, l'on conclut plus facilement à une inexécution fautive de la prestation de travail); débrayages inopinés, à des heures diverses ; défectuosités des produits fabriqués ... ${ }^{13}$.

Le droit civil québécois, on l'a vu, pourrait envisager la grève tournante sensiblement de la même façon. (Ajoutons, sans qu'il s'agisse nécessairement de grève tournante, que l'existence d'unités de négociation multiples dans une même entreprise, peut donner lieu, le cas échéant, à des arrêts de travail que l'on devrait qualifier de «partiels», envisagés à

8. De façon générale, à ce sujet, voir notamment A. NADEAU, Traité de droit civil du Québec, tome huitième. Wilson \& Lafleur (Limitée), Montréal, 1949, ch. V, pp. 195 et sq.; P. AzARD, Supplément au traité pratique de la responsabilité civile de Henri Lalou, Paris, Dalloz, 1962, no 861, p. 57 et no 878, ibidem.

9. Commissaires d'écoles pour la municipalité de Saint Tharcicius v. Association des enseignants de la Matapédia, supra note 3, à la p. 4.

10. Commission Hydroélectrique de Québec v. Filteau, (1969) D.C.R.T. 161, à la p. 162.

12. H. SINAY, op. cit., supra note 4, pp. 161 à 169.

12. A. BRUN, op. cit., supra note 4, p. 629.

13. H. Sinay, op. cit., supra note 4, p. 167. 
l'échelle de cette dernière, même s'ils mobilisent l'ensemble des salariés d'une unité de négociation.)

\section{Légalité en regard du Code du travail}

Le Code du travail réglemente l'exercice du droit de grève. La grève tournante, comme toute autre grève, se trouve soumise à des exigences générales quant au moment de son déclenchement ${ }^{14}$.

De plus, dans le cas d'un service public, l'article 99 du Code impose à l'association de salariés de donner un préavis au ministre «d'au moins huit jours » lui indiquant le moment où elle entent y recourir. Cette exigence additionnelle revêt une importance particulière dans le cas d'une grève tournante.

S'agit-il, peut-on d'abord se demander, d'un avis d'intention ou plutôt du déclenchement effectif de la grève ? Pour le juge Côté, l'avis d'intention est insuffisant ${ }^{15}$. En d'autres termes, il n'admet pas que l'association puisse décréter la grève à un moment subséquent à celui qu'indique l'avis, sans que pour autant la validité de cet avis, (ou mieux, peut-être, sa suffisance) n'en soit affectée. Pourtant, le texte même de la disposition parle $\mathrm{du}$ «moment où (l'association) entend recourir à la grève »; l'article 99 traite d'ailleurs plus loin (second et cinquième alinéas) aussi bien des effets d'une grève appréhendée que de ceux d'une grève en cours.

En second lieu, faut-il répéter l'avis dans le cas d'une grève tournante, à l'occasion de chaque arrêt particulier de travail ? Au contraire, un avis unique de l'ensemble du mouvement de grève suffit-il ? Le jugement de la Cour supérieure ne traite pas de la question. Toutefois, la conception que l'on se fait de l'article déterminera s'il doit ou non être répété. Dans la mesure, en effet, où on le considère comme un avis d'intention, l'avertissement initial suffira, car il permettra d'appréhender l'ensemble du mouvement de grève dans toutes ses manifestations.

\section{Effets sur le statut juridique de salarié}

«Personne ne cesse d'être un salarié pour l'unique raison qu'il a cessé de travailler par suite de grève ou lock-out ${ }^{16}$. Selon la dispo-

14. Code du travail, a. 40 à 46.

15. Commissaires d'écoles pour la municipalité de Saint Tharcicius v. Association des enseignants de la Matapédia, supra note 3 à la p. 6 .

16. Code du travail, a. 98. 
sition, le statut de «salarié » se trouve maintenu, indépendamment de la forme et de la légalité de la grève. Ainsi, notamment, dès qu'il y a grève, le salarié continue de jouir de la protection de l'activité syndicale offerte par le Code du travail ${ }^{17}$.

Ce lien de salariat reprend dans sa définition ${ }^{18}$ des éléments constitutifs du contrat individuel de travail classique : la prestation de travail; la rémunération en retour.

Quant à l'exigibilité, par l'employeur, de la prestation de travail, se maintient-elle dans le cas d'une grève tournante, à l'égard des salariés d'une unité de négociation qui, à un moment donné, ne se trouvent pas directement visés par l'un des arrêts de travail constitutifs d'une grève tournante ? Dans le cas de la grève tournante organisée en 1967 à l'HydroQuébec, la C.R.T. en vînt, pour sa part, à la conclusion que même le travail supplémentaire normal pouvait être exigé de ses salariés ${ }^{19}$.

Pour ce qui est, cette fois, de la rémunération dont les salariés peuvent être créanciers, il semble aller de soi, en principe, que ces derniers n'y ont droit que dans la mesure où ils ont effectivement travaillé. Exceptionnellement, il pourrait se présenter un cas de désorganisation totale de l'entreprise touchée par la grève tournante, qui y rende toute production efficace impossible. Des salariés qui auraient recherché cette désorganisation d'ensemble pourraient-ils prétendre à leur salaire, du seul fait qu'ils auraient été «disponibles » durant les intervalles de temps séparant leurs arrêts de travail formels ? La jurisprudence française a déjà rejeté cette prétention ${ }^{20}$; il s'agissait là, selon la Cour de cassation, d'une exécution du travail «dans les conditions autres que celles prévues au contrat de travail ou pratiquées dans la profession $\gg 21$.

\section{CONCLUSION}

Une telle recherche de la désorganisation de l'entreprise par les grévistes demeurant toutefois l'exception, la grève tournante paraît, en principe, licite. Les applications de ce principe, à cause de l'évolution des techniques de lutte syndicale, feront sans doute l'objet d'une étude jurisprudentielle et doctrinale plus poussée dans l'avenir.

17. Ibidem, a. 14 et suivants.

18. Ibidem, a. $1, \mathrm{~m}$.

19. Commission Hydroélectrique de Québec v. Filteau, supra note 10.

20. Hychter v. Sté des aciers Fins de l'Est, Droit social, 1965, p. 107, arrêt de la Cour de Cassation du 16 juillet 1964.

21. J. SAVATIER, commentaire de l'arrêt précité, ibidem. 